\title{
The correlation between gross domestic product per capita and the success rate of assisted reproductive technologies worldwide
}

Jie Dong ( $\sim$ dongjie8263@163.com )

The Fourth Military Medical University https://orcid.org/0000-0001-6786-1057

\section{Song Yan}

Tangdu Hospital, The Fourth Military Medical University

Chenxi Qian

Tangdu Hospital, The Fourth Military Medical University

\section{Xiaohong Wang}

Tangdu Hospital, The Fourth Military Medical University

\section{Research}

Keywords: GDP, assisted reproductive technologies, pregnancy rate, delivery rate

Posted Date: January 23rd, 2021

DOI: https://doi.org/10.21203/rs.3.rs-151639/v1

License: (c) (1) This work is licensed under a Creative Commons Attribution 4.0 International License. Read Full License

Version of Record: A version of this preprint was published at JBRA Assisted Reproduction on January 1st, 2021. See the published version at https://doi.org/10.5935/1518-0557.20210078. 


\section{Abstract \\ Background}

Although there has been increased utilization of assisted reproductive technologies (ART) in the world, there is no conclusive definition of the relationship between the success rate of ART and national wealth.

\section{Methods}

In this study, using the data from the International Committee for Monitoring Assisted Reproductive Technologies (ICMART), we sought to determine whether there is a correlation between the success rate of ART (represented by pregnancy/delivery rates) and national wealth (represented by gross domestic product per capita (GDP)). Moreover, to further understand the effect of GDP on ART effectiveness, we analyzed the association between ART success rate and GDP in 50 US states.

\section{Results}

Our data showed that the number of ART treatment cycles increased with an increase in GDP. However, we found a negative correlation between ART success rate and GDP in ICMART countries, but no correlation was seen in the US states. Using rough estimation, we derived that the success rate of ART was not related to GDP in the ICMART countries with GDP per capita more than thirteen thousand dollars.

\section{Conclusions}

In this study, for the first time, we show that when the GDP of an economic body reaches (or exceeds) thirteen thousand dollars, its ART pregnancy and delivery rates cannot be associated with GDP, and the ART success rate remains stable.

\section{Plain English Summary}

Despite the increased utilization of ART in the world, there is no conclusive definition of the relationship between the success rate of ART and national wealth. Our study utilized the data from ICMART and sought to determine whether there is a correlation between the success rate of ART and national wealth. To further understand the effect of GDP on ART effectiveness, we also analyzed the association between ART success rate and GDP in 50 US states.

We found there was a negative correlation between ART success rate and GDP in ICMART countries, but no correlation in the 50 US states. Using rough estimation, we revealed that ART success rate was not related to GDP in ICMART countries whose GDP was more than thirteen thousand dollars. Our study, for 
the first time, reveals that the success rate of ART could attain a stable level when the national or regional wealth reaches a certain level (GDP per capita of thirteen thousand dollars).

\section{Background}

Since the world's first "test-tube" baby was born in 1978, assisted reproductive technologies (ART) have been rapidly developed and widely used all over the world. The commonly used ART includes in vitro fertilization (IVF), intracytoplasmic sperm injection (ICSI), and frozen embryo transfer (FET). It has been reported that over eight million babies have been born via ART [1]. Today, in some countries, ARTconceived infants account for between $1.8-5 \%$ of all infants [2, 3]. However, despite the advances in ART, its success rate per treatment cycle remains low. Based on the ART data of 69 countries, the ICMART has reported that the delivery rate via IVF/ICSI (combined) and FET is $19.8 \%$ and $22.1 \%$, respectively [4]. In the United States, official data shows that the live-birth rate per cycle via IVF/ICSI (combined) and FET for non-donor women under 35 -years is $31.0 \%$ and $49.4 \%$, respectively; and the rate gets lower for older women [2].

Multiple factors are considered to be related to the low percentages of live births, including ages of parents, infertility related factors, and ART manipulation itself [5]. The quality and policies of a healthcare system may also influence the ART success rate. For example, a superior healthcare system that is funded properly could mean better ART services. Commonly, developed countries/regions provide superior healthcare systems and funds to support ART treatments to infertile couples. Hence, ART treatments in these countries/regions are relatively affordable, especially to sub-fertile couples, and may achieve a high success rate. Surprisingly, a study performed by Lass et al. [6] rebuts this postulation. Although the study found a robust positive correlation between the number of IVF cycles performed and GDP, it also showed that both the pregnancy and delivery rates via IVF are inversely correlated with GDP. The researchers assumed that the unexpected findings were associated with inadequate comprehensive data collection (such as age distribution, causes of infertility, and quality control level of embryo handling - due to objective limitations). Based on their limited data, however, it appears that developed countries do not have a high IVF success rate.

Despite the gradual increase in the usage of other regular ART treatments (such as ICSI and FET) [3], there has been no conclusive answer to the relationship between the success rate of ICSI/FET cycles and national wealth. Therefore, using data from ICMART, we investigated whether there is a correlation between pregnancy/delivery rate in ICSI/FET cycles and national wealth represented by GDP. Moreover, to increase the understanding of the effect of national wealth on the effectiveness of ART, we analyzed the relationship between ART success rate and GDP in 50 states of the US.

\section{Methods}

All original data analyzed were collected from published papers and materials. We used the latest ICMART ART data (including treatment cycles, pregnancy, and delivery rates) [4] and the 2012 GDP per 
capita of each country (https://www.kylc.com/stats/global/yearly/g_gdp_per_capita/2012.html). Given the potential influence of confounding factors, such as demographic and cultural characteristics, we increased our scope to analyze ART data from 50 US states. To maintain consistency with the 2012 ICMART data, we collected the 2012 ART data of individual states released by the US health department in 2015 [7], and the 2012 GDP per capita of each state [8]. The original data are populated in the Supplementary tables.

Statistical Analyses

Data analyses were performed using IBM SPSS Statistics 23.0 (IBM software, NY, USA). Graphics drawing was performed using Prism 8 (GraphPad Software, CA, USA). Normal variables are shown as average with standard deviation, and non-normal variables are shown as median with percentile values. We used Spearman correlation analysis to investigate the relationship between GDP (unit: 10 thousand dollars) and ART cycles, and employed the Pearson correlation coefficient (CC) to investigate the relationship between GDP and ART success rate after logarithmic processing of GDP (shown as LogGDP). A p-value $<0.05$ (two-tailed) was considered significant.

\section{Results}

1. The number of ART treatment cycles increased with an increase in GDP based on 2012 ICMART data

Considering the different ARTs corresponding to different applicable conditions, we analyzed the correlation between the GDP and the number of cycles of IVF, ICSI, and FET, respectively. Based on ICMART data, we calculated the median of GDP to be $1.53(0.74-4.36)$, and the median of the number of cycles of IVF, ICSI, and FET cycles to be 920.0 (277.3-3356.5), 2529.0 (631.5-8988.3), and 713.5 (118.85117.0), respectively. As shown in Fig. 1, there was a significant positive correlation between GDP and the number of ART cycles for all its various forms, including IVF (Fig. 1a, CC =0.695, $p<0.001$ ), ICSI (Fig. 1b, $\mathrm{CC}=0.644, p<0.001$ ), and FET cycles (Fig. $1 \mathrm{C}, \mathrm{CC}=0.666, p<0.001$ ). The results suggest that ART treatment cycles increase with an increase in the GDP of a country.

2. The pregnancy and delivery rates both in IVF and ICSI aspiration cycles are negatively correlated with GDP based on 2012 ICMART data.

Next, we tested the correlation between pregnancy and delivery rates in the three regular ART cycles (IVF, ICSI, and FET) and LogGDP using Pearson analysis. The results showed that in IVF cycles, both pregnancy (Fig. 2a, CC=-0.263, $p=0.041$ ) and delivery rate (Fig. $2 b, C C=-0.329, p=0.012$ ) are inversely associated with LogGDP of ICMART countries. Similarly, for ICSI cycles, there was a significant negative correlation between pregnancy (Fig. 2c, CC=-0.394, $p=0.001$ ) and delivery rate (Fig. 2d, CC=-0.417, $p=$ 0.001 ) and logGDP. However, in FET cycles, we found there was no significant correlation between pregnancy (Fig. 2e, CC=-0.006, $p=0.961$ ) and delivery rate (Fig. 2f, CC=-0.059, $p=0.565$ ) and LogGDP. The data here indicate that the success rate in IVF and ICSI cycles declines with an increase in the GDP of a country. 
3. There was no correlation between ART success rate and GDP based on the 2012 ART data of the 50 US states.

Considering the potential influence of complex demographic, geographical, and cultural backgrounds may also affect the correlation between GDP and ART success rate, we used data from 50 US states with relatively lower confounding factors to analyze the relationship between GDP and ART success rate. First, we calculated that the average pregnancy and delivery rates were $41.6 \pm 5.9 \%$ and $34.2 \pm 5.4 \%$ in ART cycles, respectively. Subsequently, we analyzed the correlation between ART cycles (excluding FET cycles) and GDP, and the results showed that ART procedures increased with an increase in GDP (Fig. 3a, $C C=0.298, p=0.036$ ). However, we found that there was no significant relationship between pregnancy (Fig. 3b, CC=-0.019, $p=0.896)$ and delivery rate (Fig. 3c, $C C=-0.078, p=0.591)$ and LogGDP.

4. There may be a boundary point to link the correlation between ART success rate and GDP.

Based on the analysis of 2012 ICMART data and 2012 ART data of 50 US states, we found that there was a negative link between ART success rate and GDP in ICMART countries, but no correlation in the US states. Because the median GDP of the US states [5.38 (4.54-6.15)] in 2012 was much higher than that of ICMART countries [1.53 (0.74-4.36)], we speculated that the ART success rate may have nothing to do with GDP when the GDP of one economic body reaches a certain high level. In other words, there may be a correlation between the ART success rate and GDP within a certain GDP range in ICMART members. To estimate the boundary point, we compared the correlative variables at different GDP levels in determining the association between the ART success rate and GDP. As shown in Table 1, when the GDP was set at $\geq$ twelve thousand dollars, we found there was a significant negative correlation between pregnancy rate only in IVF cycles and GDP (CC=-0.380, $p=0.026)$; however, when the GDP was over thirteen thousand dollars, there was no statistically significant correlation between pregnancy/delivery rate in IVF and ICSI cycles and GDP. The results indicate that there could be no correlation between ART success rate and GDP when the GDP of economic bodies is greater than thirteen thousand dollars. 
Table 1

Spearman correlation analysis between GDP per capita and ART pregnant rate and delivery rate when GDP per capita is $\geq 1.2$ or $\geq 1.3$, respectively

\begin{tabular}{|lllllll|}
\hline \multicolumn{2}{|c|}{$\begin{array}{l}\text { GDP per capita } \geq 1.2 \\
\text { (LogGDP } \geq 0.08)\end{array}$} & & & \multicolumn{3}{l|}{$\begin{array}{l}\text { GDP per capita } \geq 1.3 \\
\text { (LogGDP } \geq 0.11)\end{array}$} \\
\hline & CC & $p$ value & & CC & Mean (deviation) & $p$ value \\
\hline IVF-PR & -0.380 & 0.026 & IVF-PR & -0.320 & $31.7 \pm 6.85$ & 0.079 \\
$n=34$ & & & $n=31$ & & & \\
IVF-DR & -0.225 & 0.216 & IVF-DR & -0.133 & $23.0 \pm 7.4$ & 0.483 \\
$n=32$ & & & $n=30$ & & & \\
ICSI-PR & -0.291 & 0.089 & ICSI-PR & -0.205 & $29.3 \pm 4.6$ & 0.260 \\
$n=35$ & & & $n=32$ & & & \\
\hline ICSI-DR & -0.090 & 0.620 & ICSI-DR & 0.039 & $21.5 \pm 4.9$ & 0.834 \\
$n=33$ & & & $n=31$ & & & \\
\hline
\end{tabular}

\section{Discussion}

In this study, we investigated the correlation between the ART success rate of a country (represented by pregnancy/delivery rates) and its wealth (represented by GDP). Relying on data from 2012 ICMART members and 50 US states, we found different results: there was a negative correlation between ART success rate and GDP in ICMART countries, but no correlation in the 50 US states. Using rough estimation, we revealed that ART success rate was not related to GDP in ICMART countries whose GDP was more than thirteen thousand dollars. Therefore, we conclude that when the GDP of a country or an economic body exceeds thirteen thousand dollars, their ART pregnancy and delivery rates may remain at a stable level.

It is estimated that more than 48 million people are affected by infertility worldwide [9]. Many IVF clinics are being opened across the world to help infertile couples have babies. Data from the IVF-Worldwide website (www.ivf-worldwide.com) show that more than 3000 IVF clinics are operational, but ART treatments are still inadequate in many parts of the world, particularly in developing countries [10]. However, the prevalence of infertility worldwide is comparable across low-, middle-, and high-income countries [11]. Accordingly, wealthier countries show more utilization of ART treatments. According to our data, there is a higher ART utilization rate (including IVF, ICSI, and FET cycles) in countries/regions with high GDP per capita. In their study, Lass et al. [6] also showed a positive correlation between the utilization rate of IVF and the GDP of countries/regions. This can be attributed to the affordability of receiving ART treatments in these countries/regions. As such, governments across the world should begin and/or continue/increase support for ART treatments, as occurs in Nordic countries [6]. 
Generally, people expect developed countries/regions to have high success rates of ART treatments. Surprisingly, however, data from ICMART member countries showed a strong negative correlation between GDP and IVF/ICSI treatment success rates in developed countries/regions. Noticeably, countries with lower GDPs had higher pregnancy and delivery rates in IVF/ICSI treatments. It is difficult to determine the cause of this startling relationship owing to data availability limitations. A few potential factors may explain this relationship. For example, it is well known that female age is remarkably associated with fertility and ART success rate; women aged over 35 years have reduced fecundity and, therefore, lower pregnancy rates especially via ART treatments [12]. Evidence shows that, in developed countries, women prefer to have children late in their lives [13]. To allow time to finish their education, pursue careers, or attain financial independence, many women in developed countries delay starting families until they are 40 years or older $[13,14]$. This phenomenon means that reproductive health clinics are treating a higher number of older infertile women with more complicated gynecological diseases, such as diminished ovarian reserve, endometriosis, and adenomyosis, thereby, the reduced ART success rate in these countries. In Japan, for example, a country with a serious population aging problem [15], the pregnancy and delivery rates are much lower even by IVF (11.7\% and $8 \%$, respectively) and ICSI ( $9.8 \%$ and $6.6 \%$, respectively) (Supplementary Table 1). Another factor affecting the ART success rate may be the number of treatment cycles. As our data show, the ART treatment cycles grow as the GDP of economic bodies increases. Statistically, the success rate of ART treatments calculated from a larger number of cases should be closer to the overall rate. Thus, the pregnancy and delivery rates in IVF/ICSI cycles in lowincome countries (such as Ghana, Mali, and Nicaragua) may not adequately reflect their ART success rate owing to the relatively small number of IVF/ICSI cycles.

Additionally, the different cultural backgrounds across regions may affect the correlation between GDP and ART success rate. However, it is difficult to test and verify their role in ART success rate owing to data unavailability. Therefore, to reduce the influence of cultural backgrounds, we used data from 50 US states with relatively lower confounding factors to analyze the relationship between GDP and ART success rate. Surprisingly, we found that there was no significant correlation between GDP and ART pregnancy/delivery rate based on the US CDC data. Given the high GDP of the US states, we hypothesized that the ART success rate may have nothing to do with GDP when the GDP of one economic body reaches a certain high level. Subsequently, by analyzing data of ICMART members, we estimated this GDP level to be thirteen thousand dollars; in other words, the ART pregnancy and delivery rates were more stable (20$30 \%$, Table 1) in the regions where the GDP was more than thirteen thousand dollars. Notably, however, the success rate of ART is still not satisfactory worldwide. The latest data from the European Society of Human Reproduction and Embryology (ESHRE) show that the live-birth percentage per aspiration is $20 \%$ in both IVF and ICSI cycles [3]. It appears that the era of improving ART success rate is moving into a bottleneck period as ART has been developing for 40 years.

In this study, there exists limitations about the data quality due to the lack of basic characteristics, such as the age distribution of patients, infertile factors, and the quality control standards of reproductive health clinics. It was difficult to collect such detailed information owing to inaccessibility to the reporting registry system. Moreover, approximately only one-third of countries in the world publish their ART data to 
ICMART. Currently, a large number of reproductive health clinics are opening in Asian countries, such as China, Thailand, and Vietnam. It will be crucial to obtain ART registry data from these countries.

Additionally, owing to the limited ICMART data from a rather small number of countries, we were not able to obtain a fitting curve to show the correlation between GDP and ART success rate (data not shown). We provide only a rough estimate ( $\sim$ thirteen thousand dollars) of the boundary point. Therefore, there is a possible bias in obtaining this critical value.

\section{Conclusions}

In summary, our study, for the first time, revealed that the success rate of ART could attain a stable level when the national or regional wealth reaches a certain level (GDP per capita of thirteen thousand dollars). Despite the low number of ART treatment cycles in developing countries, the ART success rate of these countries is not inferior to that of developed countries. Nevertheless, further studies analyzing more ART data collected from more countries across the world are necessary to understand the influence of socioeconomic status on the overall effectiveness rate of ART treatment.

\section{Abbreviations}

ART

Assisted Reproductive Technologies

CC

Correlation Coefficient

FET

Frozen Embryo Transfer

GDP

Gross Domestic Product per Capita

ICSI

Intracytoplasmic Sperm Injection

ICMART

International Committee for Monitoring Assisted Reproductive Technologies

IVF

In Vitro Fertilization (IVF)

\section{Declarations}

\section{Data sharing}

All data generated in this study are included in the supplementary files.

\section{Competing interests}

The authors have no conflicts of interest to declare. 
J.D. carried out the study design, data analyzing and manuscript writing. Y. S. participated in the data collection. C. Q. performed the statistical analysis. X. W. participated in its design and helped to draft the manuscript. All authors read and approved the final manuscript.

\section{Acknowledgments and Funding}

We thank the funding supported by the grant from the National Natural Science Foundation of China (82071717). We would like to thank Editage (www.editage.cn) for English language editing.

\section{References}

1. European Society of Human Reproduction and Embryology. More than 8 million babies born from IVF since the world's first in 1978: European IVF pregnancy rates now steady at around 36 percent, according to ESHRE monitoring. ScienceDaily. Available at: www. sciencedaily.com/releases/2018/07/180703084127.htm. Accessed July 3, 2018.

2. Sunderam S, Kissin D, Zhang Y, Folger S, Boulet S, Warner L, Callaghan W, Barfield W: Assisted Reproductive Technology Surveillance - United States, 2016.Morbidity and mortality weekly report Surveillance summaries (Washington, DC : 2002) 2019, 68:1-23.

3. Wyns C, Bergh C, Calhaz-Jorge C, De Geyter C, Kupka M, Motrenko T, Rugescu I, Smeenk J, TandlerSchneider A, Vidakovic S, Goossens V: ART in Europe, 2016: results generated from European registries by ESHRE.Human reproduction open 2020, 2020:hoaa032.

4. de Mouzon J, Chambers G, Zegers-Hochschild F, Mansour R, Ishihara O, Banker M, Dyer S, Kupka M, Adamson G: International Committee for Monitoring Assisted Reproductive Technologies world report: assisted reproductive technology 2012t. Human reproduction (Oxford, England) 2020, 35:1900-1913.

5. Tarín J, García-Pérez M, Cano A: Assisted reproductive technology results: why are live-birth percentages so low? Molecular reproduction and development 2014, 81:568-583.

6. Lass A, Chaudary W, Lass G: Correlation between gross domestic product, utilization of in vitro fertilization, and pregnancy success rate across the world.Journal of medical economics 2019, 22:878-882.

7. Sunderam S, Kissin D, Crawford S, Folger S, Jamieson D, Warner L, Barfield W: Assisted Reproductive Technology Surveillance - United States, 2012.Morbidity and mortality weekly report Surveillance summaries (Washington, DC : 2002) 2015, 64:1-29.

8. Brooks LR, Cao LX, Rodriguez RM: Gross Domestic Product by State Advance Statistics for 2012 and Revised Statistics for 2009-2011.Survey of Current Business 2013.

9. Mascarenhas MN, Flaxman SR, Boerma T, Vanderpoel S, Stevens GA: National, regional, and global trends in infertility prevalence since 1990: a systematic analysis of 277 health surveys. PLOS Med 2012, 9:e1001356. 
10. Inhorn MC, Patrizio P: Infertility around the globe: new thinking on gender, reproductive technologies and global movements in the 21st century. Hum Reprod Update 2015, 21:411-426.

11. Asemota OA, Klatsky P: Access to infertility care in the developing world: the family promotion gap.Semin Reprod Med 2015, 33:17-22.

12. Howles $\mathrm{CM}$, Kim CH, Elder $\mathrm{K}$ : Treatment strategies in assisted reproduction for women of advanced maternal age.Int Surg 2006, 91:S37-54.

13. Mills $M$, Rindfuss RR, McDonald P, te Velde E: Why do people postpone parenthood? Reasons and social policy incentives. Hum Reprod Update 2011, 17:848-860.

14. Armstrong $\mathrm{S}$, Akande $\mathrm{V}$ : What is the best treatment option for infertile women aged $\mathbf{4 0}$ and over? $\mathrm{J}$ Assist Reprod Genet 2013, 30:667-671.

15. Nishi N, Ikeda N, Sugiyama T, Kurotani K, Miyachi M: Simulating the Impact of Long-Term Care Prevention Among Older Japanese People on Healthcare Costs From 2020 to 2040 Using System Dynamics Modeling.Front Public Health 2020, 8:592471.

\section{Figures}
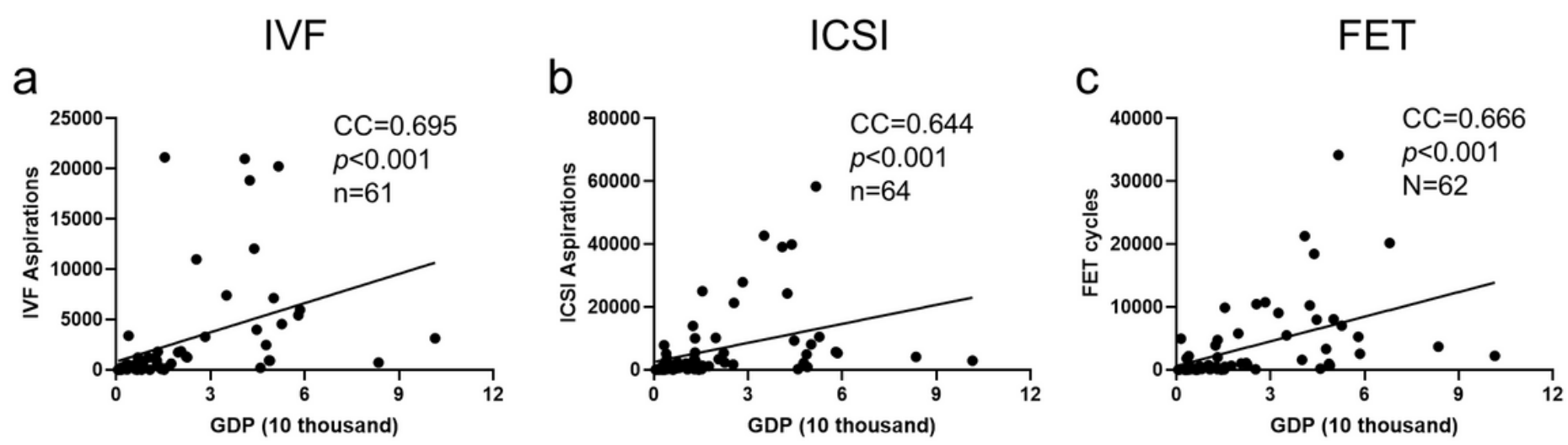

Figure 1

The correlation between GDP (10 thousand dollars) and numbers of ART treatment cycles from 2012 ICMART data. a: correlation between GDP and IVF cycles; b: correlation between GDP and ICSI cycles; c: correlation between GDP and FET cycles. CC: correlation coefficient. 


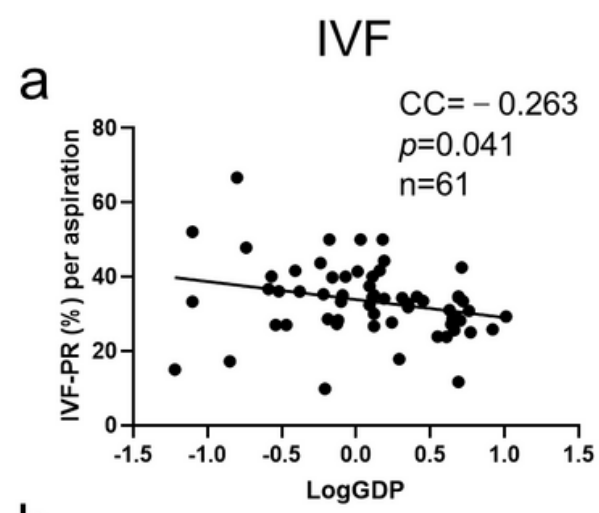

b

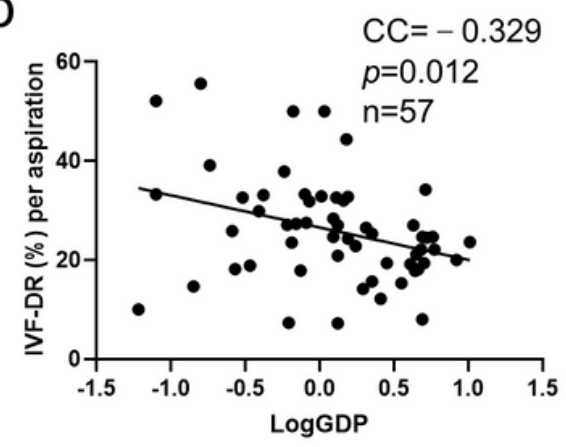

ICSI

C

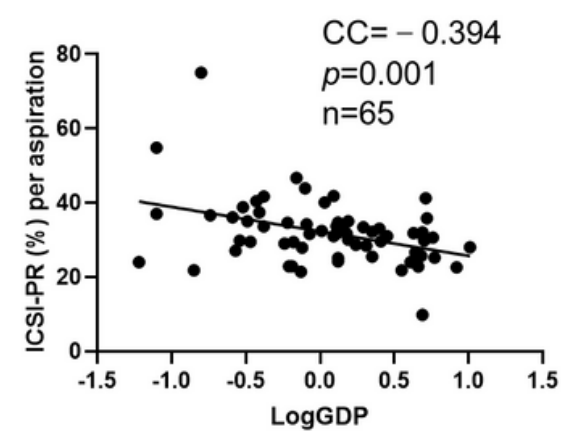

d

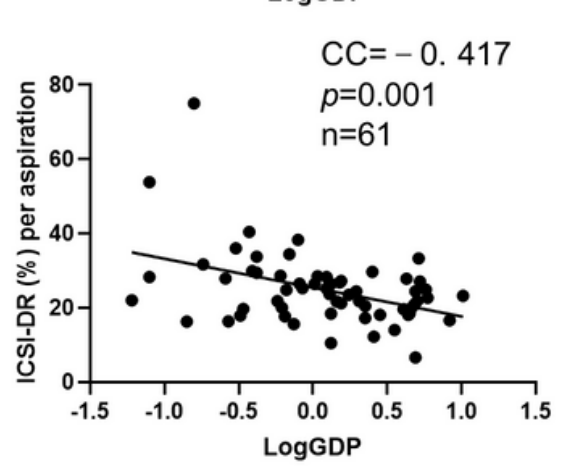

FET

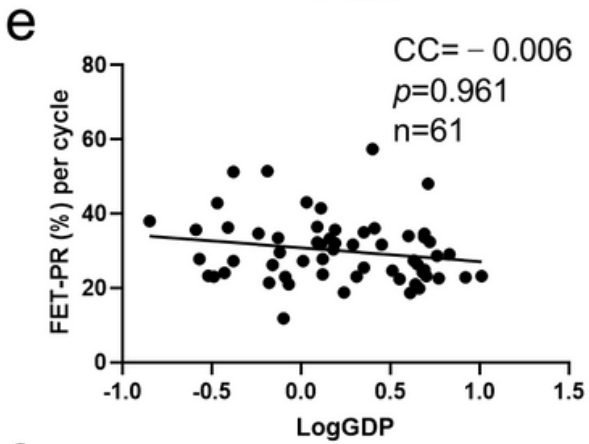

$f$

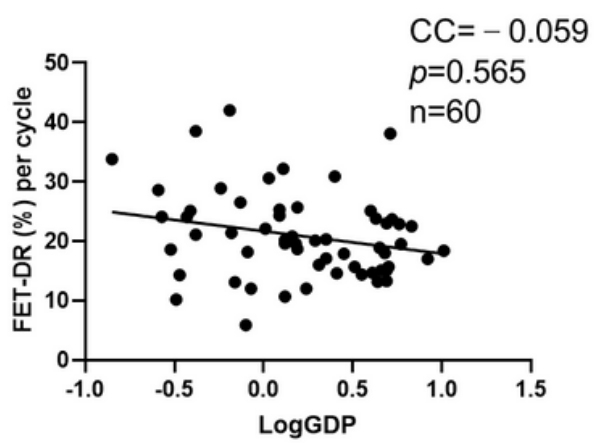

Figure 2

The correlation between LogGDP and pregnancy/delivery rate in ART cycles from 2012 ICMART data, respectively. a: pregnancy rate in IVF cycles (IVF-PR). b: delivery rate in IVF cycles (IVF-DR); c: pregnancy rate in ICSI cycles (ICSI-PR). d: delivery rate in ICSI cycles (ICSI-DR); e: pregnancy rate in FET cycles (FETPR). f: delivery rate in FET cycles (FET-DR). CC: correlation coefficient.
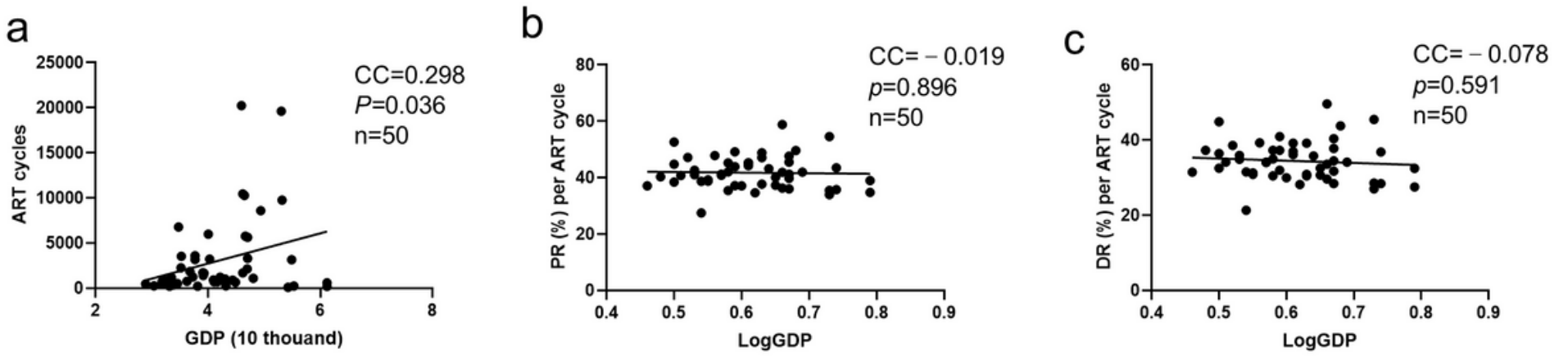

Figure 3

The correlation between GDP and numbers of ART treatment cycles from 2012 USA data (a). The correlation between LogGDP and pregnancy rate (b) and delivery rate (c) in ART cycles, respectively. CC: correlation coefficient.

\section{Supplementary Files}

This is a list of supplementary files associated with this preprint. Click to download. 
- Supplymentarytables.docx

Page $12 / 12$ 